\title{
A fast and effective method to perform paternity testing for Wolong giant pandas
}

\author{
LI DeSheng ${ }^{1,3}$, CUI HengMin ${ }^{2}$, WANG ChengDong ${ }^{1}$, LING ShanShan ${ }^{1}$, HUANG Zhi ${ }^{1}$ \& \\ ZHANG HeMin ${ }^{1 *}$ \\ ${ }^{1}$ China Research and Conservation Center for the Giant Panda, Ya'an 625000, China; \\ ${ }^{2}$ Medical College of Animal, Sichuan Agricultural University, Ya'an 625000, China; \\ ${ }^{3}$ Key Laboratory of Conservation Biology for Endangered Wildlife of the Ministry of Education and State Conservation Center for Gene Re- \\ sources of Endangered Wildlife, Hangzhou 310058, China
}

Received March 1, 2011; accepted April 27, 2011

\begin{abstract}
Captive populations provide a precious genetic resource for endangered animals and a source of individuals for reintroduction to depleted habitats. Therefore, accuracy in determining paternity is of vital importance for managing captive populations and in selecting representative individuals of known genetic characteristics for release. In this study, we established a fast and effective method to conduct paternity testing for captive giant pandas in the Wolong population. This technique uses two highly polymorphic microsatellites initially, subsequent use of five less polymorphic markers and then paternity exclusion testing carried out using the giant panda paternity exclusion program we have developed. Our results revealed that (1) both sets of markers successfully identified the real fathers in 25 cases of paternity testing and (2) the success rate of paternity exclusion varied with the degree of polymorphism of the markers used. Subsequently, we conducted correlation analysis between the success rates of paternity identification with these markers, parameters of genetic diversity and tests of neutrality. We found that the paternity exclusion power of microsatellites was significantly correlated with the number of alleles $(\mathrm{Na})$, expected heterozygosity $\left(H_{\mathrm{E}}\right)$ and observed homozygosity statistic $\left(F_{\mathrm{O}}\right)($ all $P<0.05)$. From this, we developed a new variable, $N \mathrm{a} \times H_{\mathrm{E}} / F_{\mathrm{O}}$, showing a highly significant positive correlation with the resolution power of microsatellites $(P=0.001)$. Moreover, the first two highly polymorphic loci gave a $100 \%$ success rate of excluding non-paternal males because they yielded higher values of $N \mathrm{a} \times H_{\mathrm{E}} / F_{\mathrm{O}}$ than the other five less polymorphic markers. Thus, the $N \mathrm{a} \times H_{\mathrm{E}} / F_{\mathrm{O}}$ parameter appears suitable to serve as a criterion for selecting microsatellite markers, which could be used for high-resolution molecular techniques of paternity determination among a range of captive animals besides giant pandas.
\end{abstract}

giant panda, paternity testing, microsatellite, correlation

Citation: $\quad$ Li D S, Cui H M, Wang C D, et al. A fast and effective method to perform paternity testing for Wolong giant pandas. Chinese Sci Bull, 2011, 56: 2559-2564, doi: 10.1007/s11434-011-4571-3

The biological diversity of the planet is being depleted rapidly as a direct and indirect consequence of human actions [1]. Many species are already extinct and the populations of many others have been reduced to levels where they risk extinction. The International Union for the Conservation of Nature (IUCN) 2011 Red List shows that a total of 10359 animal species are classified into the categories of Extinct (EX), Extinct in the Wild (EW), Critically Endangered (CR),

*Corresponding author (email: hmhm_zhang@163.com)
Endangered (EN) and Vulnerable (VU) (http://www. iucnredlist.org). Out of these species, 4831 are listed in the EW, CR and EN appendixes and require captive breeding programs to preserve their gene resources and protect them. For the EW animals, captive breeding is the only way to ensure survival of their species. For the CR and EN animals, captive populations are not only precious genetic resources but also serve as sources of individuals that can be released for reintroduction projects. Therefore, scientific managment of captive populations is of importance for the conservation 
of endangered animals.

Artificial insemination (AI) can be used to (1) control the genetic contribution of individuals that are unable to breed naturally, (2) enhance the reproductive rate of males of high genetic quality and (3) increase the overall success rate of reproduction. To expand captive populations quickly, the AI technique has been used in artificial reproduction of the cheetah [2], black-footed ferret [3], pampas deer [4], and other endangered animals [5]. Despite the use of multiple AI or combined utilization of AI and natural mating (NM) contributed partly to the relatively large captive population, but it also introduced a problem of mating between multiple males and single female to studbook records, thus making that genetic tool is the only choice to get a pedigree for those AI offspring.

The giant panda (Ailuropoda melanoleuca) is one of the most endangered animals and its captive and wild populations currently possess 293 individuals [6] and 1596 ones [7], respectively. Due to male infertility and absence of males in a facility, zoo managers usually employ sperm from several males to perform AI to ensure reproduction of captive giant pandas [8]. Thus, it is essential for managers to perform paternity testing for captive giant pandas.

Different kinds of molecular marker systems were involved in paternity determination, including DNA fingerprinting [8,9], amplified fragment length polymorphism (AFLP) [10,11] and microsatellites [12-14]. The microsatellites were recommended to serve as a preferred marker system due to their codominant inheritance, wide genome coverage, high variability and ease scoring [15]. To develop a reliable marker system to conduct paternity testing, we isolated 16 microsatellite loci for the giant panda in a previous study [16]. However, we were confused about priority of these microsatellite markers when carrying out paternity tests. Zane et al. [15,17] emphasized that the statistical power of microsatellite markers depended on the number of loci used, the degree of polymorphism of each locus and the sample size, suggesting that the microsatellites should be used in the order of polymorphism of markers.

Of the present study, our first objective is to determine the paternity issues of AI-derived giant panda cubs born in the years of 2006, 2007 and 2008 through microsatellite genotyping. The second objective is to formulate a new criterion for priority of employing microsatellite markers based on the success rate of paternity exclusion (PE) of each locus. The third objective is to develop a fast reliable method to perform paternity tests for Wolong giant pandas in the future.

\section{Materials and methods}

\subsection{Sample collection and DNA extraction}

In total, 47 fresh blood samples were collected from the China Research and Conservation Center for the Giant
Panda (Wolong) during routine medical examinations, including all of adult giant pandas and their cubs derived from 25 multiple-to-one AI cases of 2006, 2007 and 2008. Blood was collected using ethylenediaminetetraacetic acid (EDTA)containing syringes and stored at $-80^{\circ} \mathrm{C}$. Genomic DNA was extracted from blood samples using standard phenol/chloroform methods [18].

\subsection{Microsatellite genotyping}

Sixteen pairs of primers were obtained from our previous study [16]. A M13 tail (5'-CACGACGTTGTAAAACGAC-3') was added to the each forward primer to allow fluorescent labeling during amplification reactions. We performed PCR amplifications as described by Zhang et al. [16] and finally selected seven microsatellite loci yielding stable PCR products and easily scored bands in automatic genotyping to carry out paternity testing. Amplification products, loaded on $6.5 \%$ denaturing polyacrylamide gels, were analyzed on a Li-Cor 4200 automated DNA sequencer, with a size standard (50-350 bp, IRD-700). SAGA ${ }^{\mathrm{GT}}$ version 3.2 software (LI-COR) was used to analyze gel images and size the alleles accurately.

\subsection{Data collection and analysis}

Genotypic data were generated by SAGA ${ }^{\mathrm{GT}}$ version 3.2 and some manual adjustments were made based on the banding patterns on the gel images. Population genetic parameters such as the number of alleles per locus $(\mathrm{Na})$, observed heterozygosity $\left(H_{\mathrm{O}}\right)$ and expected heterozygosity $\left(H_{\mathrm{E}}\right)$ were estimated with GENETIX 4.05 [19]. Hardy-Weinberg equilibrium (HWE) was analyzed using GENEPOP 3.3 version [20]. Parameters of test of neutrality such as observed homozygosity statistic $\left(F_{\mathrm{O}}\right)$, expected homozygosity statistic $\left(F_{\mathrm{E}}\right)$ and normalized deviate of the homozygosity $(F \mathrm{nd})$ were calculated using PyPop 0.7.0 [21,22]. PE analysis was performed in GPPE (giant panda paternity exclusion) program, which was Excel-based and developed by ourselves. Correlation analyses between success rates of PE and parameters of $N \mathrm{a}, H_{\mathrm{O}}, H_{\mathrm{E}}, F_{\mathrm{O}}, F_{\mathrm{E}}$ and $F$ nd were carried out using SPSS 16.0 [23].

\section{Results and discussion}

\subsection{Genetic diversity}

The level of genetic variation was assessed by three measures, including $N \mathrm{a}, H_{\mathrm{O}}$ and $H_{\mathrm{E}}$. These seven microsatellite loci showed a total of 57 alleles, ranging from 4 to 13 alleles per locus (average 8.143) (Table 1) and exhibited the ranges of $H_{\mathrm{O}}$ and $H_{\mathrm{E}}$ as $0.575-0.915$ (average 0.745) and $0.676-0.893$ (average 0.782), respectively (Table 2). The loci Aime-14 and Aime-5 deviated from HWE and presented significantly lower $H_{\mathrm{O}}$ than $H_{\mathrm{E}}$ (Table 2). Previous studies 
Table 1 Allele frequency of seven microsatellite markers of the Wolong giant panda population ${ }^{\text {a) }}$

\begin{tabular}{|c|c|c|c|c|c|c|c|c|c|c|c|c|c|c|}
\hline Marker & $\mathrm{Na}$ & A1 & A2 & A3 & A4 & A5 & A6 & A7 & A8 & A9 & A 10 & A11 & A12 & A13 \\
\hline Aime-11 & 13 & 0.149 & 0.064 & 0.064 & 0.106 & 0.149 & 0.011 & 0.032 & 0.053 & 0.138 & 0.032 & 0.181 & 0.011 & 0.011 \\
\hline Aime-13 & 12 & 0.053 & 0.096 & 0.096 & 0.085 & 0.011 & 0.085 & 0.074 & 0.223 & 0.096 & 0.117 & 0.032 & 0.032 & \\
\hline Aime-10 & 9 & 0.032 & 0.011 & 0.032 & 0.021 & 0.468 & 0.106 & 0.255 & 0.043 & 0.032 & & & & \\
\hline Aime-3 & 7 & 0.053 & 0.245 & 0.223 & 0.128 & 0.149 & 0.117 & 0.085 & & & & & & \\
\hline Aime-14 & 6 & 0.223 & 0.170 & 0.117 & 0.096 & 0.021 & 0.372 & & & & & & & \\
\hline Aime-5 & 4 & 0.117 & 0.287 & 0.468 & 0.128 & & & & & & & & & \\
\hline
\end{tabular}

a) The bold numbers indicate the predominant allele of each marker.

revealed the levels of genetic diversity in other bears were about $2.00-8.75$ for $N a, 0.30-0.82$ for $H_{\mathrm{E}}$ [24]. For wild giant pandas, the levels of genetic variation were 3.98 for $\mathrm{Na}$ and 0.53 for $H_{\mathrm{E}}$ [24]. Therefore, the allelic polymorphism and heterozygosity showed that the captive giant pandas in Wolong possessed a relatively high level of genetic variability and were a good source for reintroduction.

\subsection{Test of neutrality}

Allele frequencies of seven microsatellite loci showed that each locus produced an obviously predominant allele and several ambiguous low-frequency alleles (Table 1). Nonetheless, the frequencies of predominant alleles of seven loci lacked correlation with the total number of alleles $(P>$ $0.05)$. Thus, the data of allele frequency were subjected to assessment of test of neutrality. The results revealed that there were three loci under expectation of neutrality, including Aime-10, Aime-16 and Aime-5 ( $P>0.05$; Table 2). To the contrary, other four loci yielded significantly deviations from $F_{\mathrm{E}}(P<0.05$; Table 2$)$, showing a more even distribution of alleles than expected from neutrality.

\subsection{Paternity testing}

According to the order of polymorphism (i.e. $\mathrm{Na}$ ), the seven microsatellite markers were used one-by-one to perform PE analysis of 25 AI cases of Wolong giant pandas (Table 3).
The results showed that (1) PE percentages of seven loci ranged from $28 \%$ to $76 \%$, (2) two most polymorphic loci Aime-11 and Aime-13 produced 100\% success rates of PE, and (3) combined utilization of other less polymorphic markers also successfully identified the real sires of 25 giant panda cubs. As a result, this study built two sets of microsatellite markers (bi- and penta-locus), which attained the identical result of paternity testing and resolved the undetermined AI issues of 2006, 2007 and 2008 in the Wolong captive population.

\subsection{Correlation analysis between $\mathrm{PE}$ percentages and other parameters}

Correlation analysis between success rates of PE and other parameters could produce an indicator for selection of molecular markers. Correlation analysis between PE percentages and $\mathrm{Na}$ values gave an expected significant positive correlation ( $P=0.017$; Table 2$)$. Moreover, our study revealed another two stronger correlates, $H_{\mathrm{E}}$ and $F_{\mathrm{O}}$ (Table 2). The $H_{\mathrm{E}}$ values showed a highly positive correlation to the success rates of $\mathrm{PE}(P=0.011$; Table 2$)$ while the $F_{\mathrm{O}}$ measures presented a significant negative correlation to $\mathrm{PE}$ percentages $(P=0.011$; Table 2$)$. Thus, we deduced a new variable $N \mathrm{a} \times H_{\mathrm{E}} / F_{\mathrm{O}}$, which produced a highly significant positive correlation to the PE power of microsatellites $(P=$ 0.001; Table 2). Furthermore, our results demonstrated that the reason that the bi-locus marker system gave a $100 \%$

Table 2 Parameters of genetic diversity $\left(\mathrm{Na}, H_{\mathrm{O}}\right.$ and $\left.H_{\mathrm{E}}\right)$ and test of neutrality $\left(F_{\mathrm{O}}, F_{\mathrm{E}}\right.$ and $F$ nd) of seven microsatellite markers ${ }^{\text {a) }}$

\begin{tabular}{|c|c|c|c|c|c|c|c|c|c|c|}
\hline Locus & $\mathrm{Na}$ & $H_{\mathrm{O}}$ & $H_{\mathrm{E}}$ & $P$ & $F_{\mathrm{O}}$ & $F_{\mathrm{E}}$ & $F$ nd & $P$ & $\%$ & $N \mathrm{a} \times H_{\mathrm{E}} / F_{\mathrm{O}}$ \\
\hline Aime-11 & 13 & 0.872 & 0.889 & 0.196 & 0.121 & 0.203 & -1.229 & $0.026^{*}$ & 76 & 95.512 \\
\hline Aime-13 & 12 & 0.872 & 0.893 & 0.234 & 0.116 & 0.222 & -1.402 & $0.004^{*}$ & 76 & 92.379 \\
\hline Aime-10 & 9 & 0.681 & 0.707 & 0.682 & 0.301 & 0.296 & 0.046 & 0.618 & 28 & 21.140 \\
\hline Aime-3 & 7 & 0.915 & 0.837 & 0.275 & 0.172 & 0.374 & -1.565 & $0.002^{*}$ & 36 & 34.064 \\
\hline Aime-14 & 6 & 0.660 & 0.767 & $0.023^{*}$ & 0.241 & 0.423 & -1.291 & $0.038^{*}$ & 44 & 19.095 \\
\hline Aime-5 & 4 & 0.575 & 0.676 & $0.016^{*}$ & 0.332 & 0.567 & -1.363 & 0.052 & 32 & 8.145 \\
\hline$r$ & 0.836 & 0.647 & 0.869 & - & -0.869 & -0.730 & -0.167 & - & - & 0.960 \\
\hline$P$ & $0.017^{*}$ & 0.116 & $0.011^{*}$ & - & $0.011^{*}$ & 0.063 & 0.355 & - & - & $0.001^{*}$ \\
\hline
\end{tabular}

a) Correlation coefficients $(r)$ between percentage of paternity exclusion $(\%)$ and other parameters were listed. *, $P$ values of significance smaller than 0.05 . 
success rate of excluding non-father males was that the two loci yielded obviously higher values of $N \mathrm{a} \times H_{\mathrm{E}} / F_{\mathrm{O}}$ than the combination of other five markers (92.379-95.512 vs. 8.145-34.964; Table 2). Therefore, we recommended the $N \mathrm{a} \times H_{\mathrm{E}} / F_{\mathrm{O}}$ parameter to serve as a new criterion for priorly employing microsatellite markers when developing highresolution molecular techniques to conduct paternity determination for captive animals.

\subsection{Establishment of a fast effective method for paternity determination}

The Li-Cor 4200 automated DNA sequencer could generate gel images, which allows us to adjust some genotyping errors from automatic scoring. Furthermore, the banding patterns of parents and offspring were present in the same gel image, which were read like DNA fingerprinting and thus provided reliable PE results. Taking Aime-11 as an example (Figure 1), the lanes No. 1, 3 and 6 were three potential fa- thers (Studbook numbers: 424, 503 and 467) while the No. 16 and No. 17 lanes were the giant panda cub (No. 652) and the mother (No. 382), respectively. The gel-based PE analysis easily identified the male of No. 424 as the real father of No. 652 (Figure 1 and Table 3).

Although there are some types of software, such as Cervus [25], FaMoz [26] and EasyPA [27], able to deal with paternity testing, these programs were designed based on maximum likelihood and/or Bayesian inference. Since the paternity issues of captive giant pandas usually involves 2-4 males, direct PE analysis is enough for identifying the real sires from AI cases, which is more accurate than likelihood and Bayesian methods. Hence, we designed an Excel-based GPPE program to conduct PE analysis of captive giant pandas. Taking cubs No. 631 and 632 as examples, we entered genotypic data of five adult and two young giant pandas into GPPE and returned that the male No. 503 was the real father of both cubs (Figure 2 and Table 3). Moreover, the results revealed that the first bi-locus marker system had

Table 3 Results of paternity testing for the giant pandas born in the years of 2006, 2007 and 2008 of the Wolong population ${ }^{\text {a) }}$

\begin{tabular}{|c|c|c|c|c|c|c|c|c|c|c|c|c|}
\hline Year & Offspring & Sex & Dam & Potential male & Sire identified & \multicolumn{7}{|c|}{ Aime- } \\
\hline \multirow{14}{*}{2006} & & & & & & $\begin{array}{c}11 \\
(13)\end{array}$ & $\begin{array}{c}13 \\
(12)\end{array}$ & $\begin{array}{l}10 \\
(9)\end{array}$ & $\begin{array}{c}3 \\
(7)\end{array}$ & $\begin{array}{l}14 \\
(6)\end{array}$ & $\begin{array}{l}16 \\
(6)\end{array}$ & $\begin{array}{c}5 \\
\text { (4) }\end{array}$ \\
\hline & 631 & $\mathrm{~F}$ & 476 & $357,369,502,503$ & 503 & $\sqrt{ }$ & $\sqrt{ }$ & $\bullet$ & $\bullet$ & $\bullet$ & & \\
\hline & 632 & $\mathrm{~F}$ & & & 503 & & $\sqrt{ }$ & $\bullet$ & $\bullet$ & $\bullet$ & & \\
\hline & 633 & $\mathrm{M}$ & 511 & $369,386,424,503$ & 424 & $\sqrt{ }$ & $\sqrt{ }$ & $\bullet$ & $\bullet$ & $\circ$ & $\circ$ & $\circ$ \\
\hline & 636 & $\mathrm{M}$ & 477 & $357,369,502,503$ & 503 & $\sqrt{ }$ & $\sqrt{ }$ & $\bullet$ & $\bullet$ & $\bullet$ & & \\
\hline & 638 & $\mathrm{~F}$ & 516 & 467,503 & 503 & & $\sqrt{ }$ & $\sqrt{ }$ & $\sqrt{ }$ & & $\sqrt{ }$ & \\
\hline & 639 & $\mathrm{M}$ & & & 503 & & $\sqrt{ }$ & & $\sqrt{ }$ & & $\sqrt{ }$ & \\
\hline & 641 & $\mathrm{~F}$ & 432 & $467,502,503$ & 502 & $\sqrt{ }$ & $\sqrt{ }$ & & & $\sqrt{ }$ & $\sqrt{ }$ & \\
\hline & 642 & M & 495 & $369,467,502,503$ & 502 & $\bullet$ & $\bullet$ & & & $\sqrt{ }$ & & \\
\hline & 643 & $\mathrm{~F}$ & & & 369 & & $\sqrt{ }$ & & $\sqrt{ }$ & $\sqrt{ }$ & & $\sqrt{ }$ \\
\hline & 651 & $\mathrm{~F}$ & & & 424 & $\sqrt{ }$ & & $\bullet$ & $\bullet$ & $\circ$ & $\circ$ & $\circ$ \\
\hline & 652 & $\mathrm{~F}$ & 382 & $424,467,503$ & 424 & $\sqrt{ }$ & $\sqrt{ }$ & $\bullet$ & $\bullet$ & & & $\sqrt{ }$ \\
\hline & 654 & $\mathrm{~F}$ & 504 & $369,424,467,479$ & 479 & $\sqrt{ }$ & $\sqrt{ }$ & $\bullet$ & $\bullet$ & $\bullet$ & $\bullet$ & \\
\hline & 664 & $\mathrm{~F}$ & 403 & 467,479 & 479 & $\sqrt{ }$ & $\sqrt{ }$ & $\sqrt{ }$ & $\sqrt{ }$ & & $\sqrt{ }$ & \\
\hline \multirow{6}{*}{2007} & 668 & $\mathrm{M}$ & 487 & $467,479,503$ & 503 & $\sqrt{ }$ & & $\sqrt{ }$ & $\bullet$ & $\bullet$ & $\bullet$ & \\
\hline & 669 & $\mathrm{~F}$ & & & 503 & $\sqrt{ }$ & $\sqrt{ }$ & $\bullet$ & $\bullet$ & $\circ$ & $\circ$ & \\
\hline & 682 & $\mathrm{~F}$ & 477 & $424,467,503$ & 424 & $\sqrt{ }$ & & $\bullet$ & $\bullet$ & & & $\sqrt{ }$ \\
\hline & 687 & $\mathrm{~F}$ & 516 & $479,503,542$ & 479 & $\sqrt{ }$ & & $\sqrt{ }$ & $\sqrt{ }$ & $\sqrt{ }$ & & $\sqrt{ }$ \\
\hline & 688 & M & & & 479 & $\sqrt{ }$ & $\sqrt{ }$ & & $\sqrt{ }$ & $\sqrt{ }$ & & $\sqrt{ }$ \\
\hline & 692 & $\mathrm{~F}$ & 474 & 467,502 & 502 & $\sqrt{ }$ & $\sqrt{ }$ & $\sqrt{ }$ & & $\sqrt{ }$ & $\sqrt{ }$ & \\
\hline \multirow{3}{*}{2008} & 706 & $\mathrm{~F}$ & 382 & 424,479 & 424 & $\sqrt{ }$ & $\sqrt{ }$ & & & $\sqrt{ }$ & & \\
\hline & 721 & M & 432 & 502,503 & 503 & & $\sqrt{ }$ & & & $\sqrt{ }$ & & \\
\hline & 735 & F & & & 488 & $\sqrt{ }$ & $\sqrt{ }$ & $\sqrt{ }$ & $\sqrt{ }$ & $\sqrt{ }$ & $\sqrt{ }$ & $\sqrt{ }$ \\
\hline \multirow{2}{*}{\multicolumn{6}{|c|}{ Percentage of paternity exclusion $(\%)$}} & 76 & 76 & 28 & 36 & 44 & 32 & 32 \\
\hline & & & & & & \multicolumn{2}{|c|}{100} & \multicolumn{5}{|c|}{100} \\
\hline
\end{tabular}

a) The numbers in parentheses were the number of alleles $(\mathrm{Na})$. Symbol " $\sqrt{ }$ " represents that this microsatellite alone is capable of excluding non-father males while symbols "•" and "○" shows that these two suites of markers also are able to identify the real sire. 


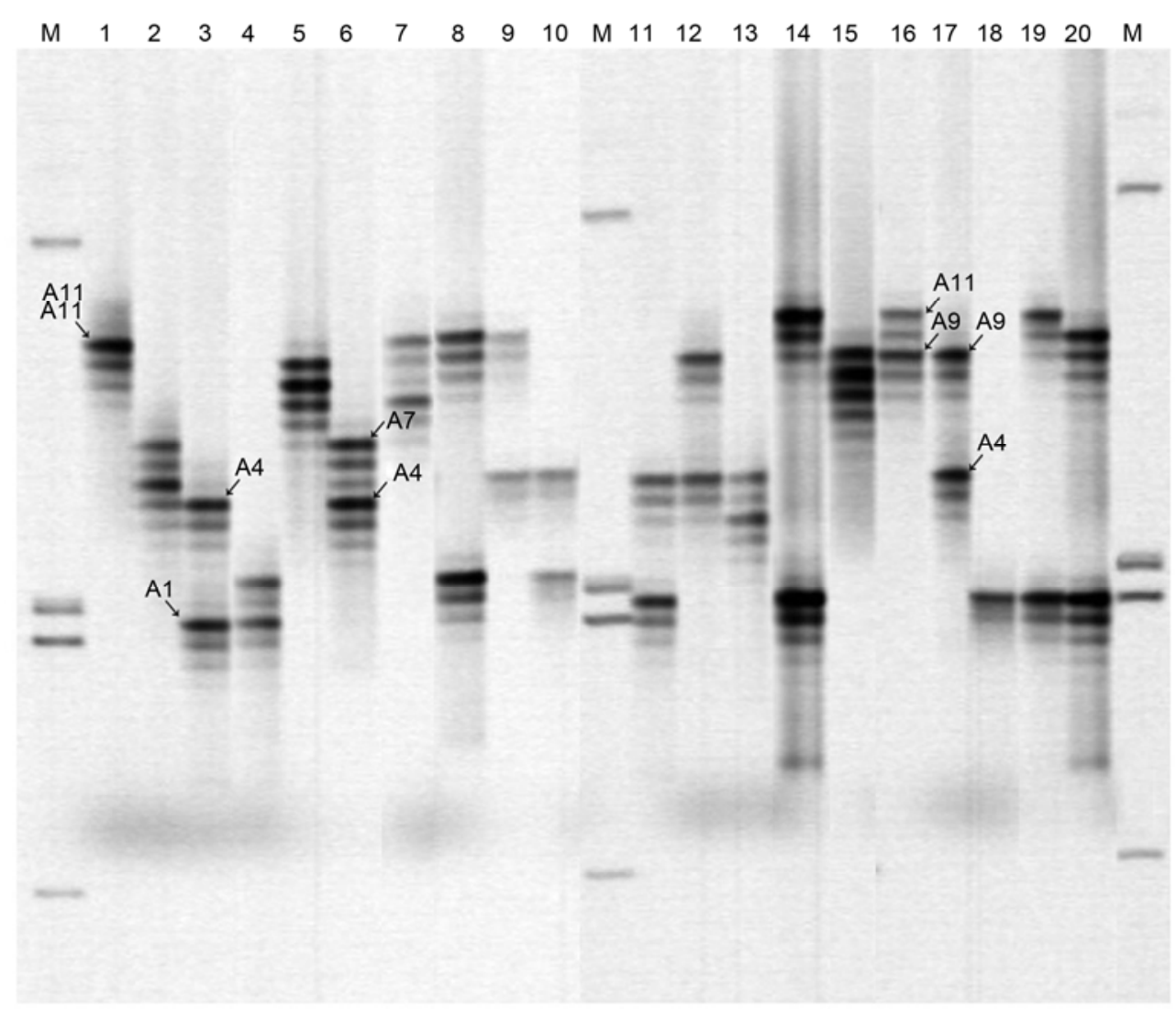

Figure 1 Genotyping gel image of Aime-11. Lanes 1-20 represent different individuals, of which the lanes 1, 3, 6, 16 and 17 were giant pandas No. 424, 503, 467, 652 and 382, respectively. M and A were abbreviations of marker and alleles. Numbering of alleles was the same as that in Table 1.

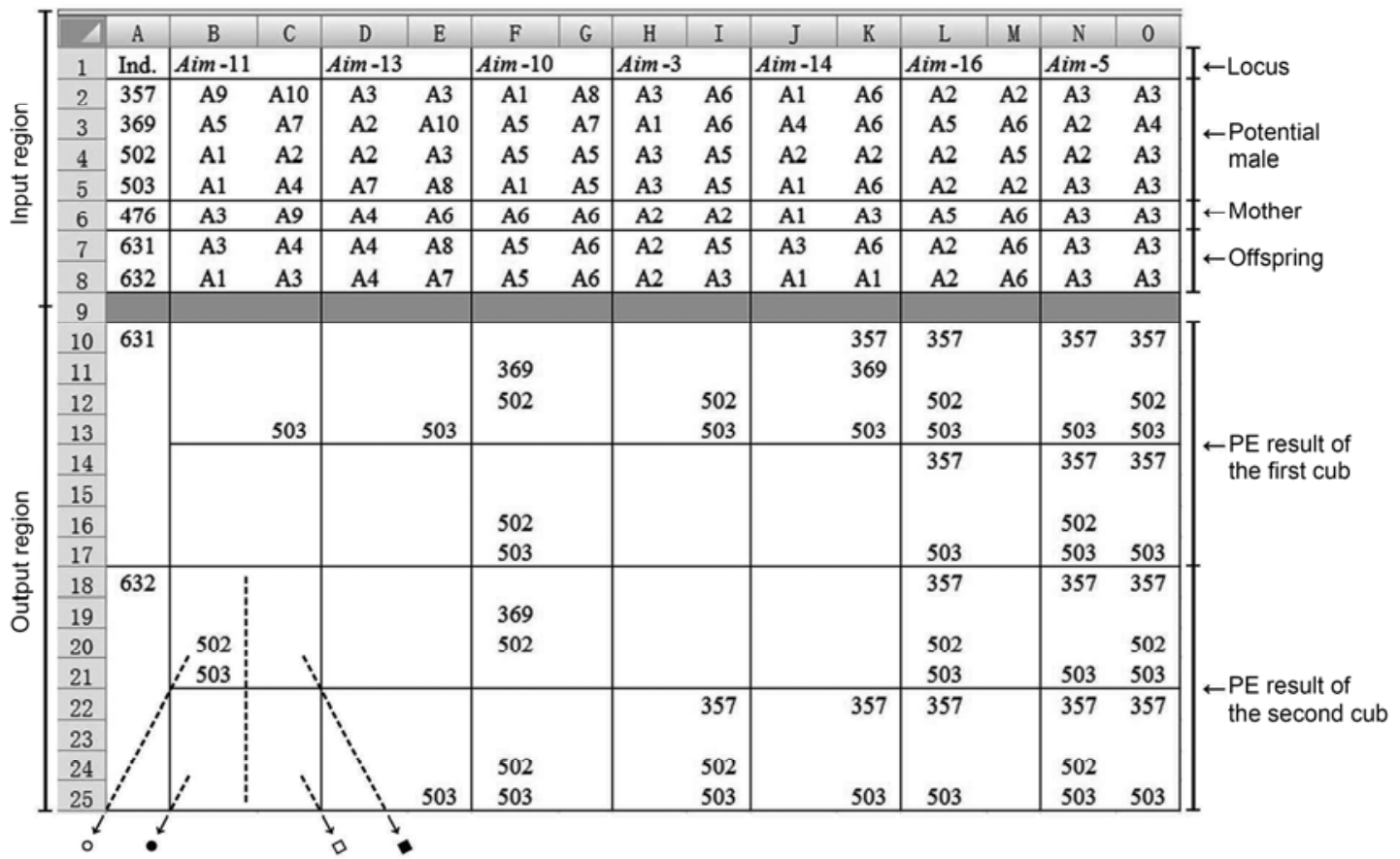

Figure 2 Interface of PE analysis of panda cubs No. 631 and 632 in GPPE program. "A" was abbreviation of allele and its numbering of each locus was the same as that in Table 1. Genotypic data of the individuals could be divided into the left and right alleles so that we designated "using the left alleles of young baby and the right alleles of adult pandas to conduct PE analysis" as "left-to-right PE". According to this rule, symbols of “०”, "•”, “ $\square$ " and "ø" represent the exclusion results of left-to-left, left-to-right, right-to-right and right-to-left, respectively. 
an obviously better exclusion power than the second penta-locus system (Figure 2 and Table 3 ). In addition, for each giant panda had a Chinese house name, for the convenience of breeders, we could easily replace studbook numbers in GPPE with Chinese house names, thus greatly facilitating management of captive giant pandas.

In conclusion, we established a fast effective method to conduct paternity tests for Wolong giant pandas; this technique includes prior genotyping of two most polymorphic microsatellite loci in a gel-based Li-Cor DNA analyzer, supplement genotyping of the penta-locus marker system and final PE analysis in GPPE program. In most AI cases, the bi-locus marker system is enough to resolve paternity issues and thus the second penta-locus technique would be used occasionally. As a consequence, this study developed a fast, reliable and economic genotyping technique for Wolong giant pandas that will be useful in the future.

We are grateful to Dr. Wan $Q H$ for her aid in the design of the GPPE program and manuscript preparation. We also thank Dr. Ni $X W$ for his help in microsatellite genotyping. This work was supported by the National Basic Research Program of China (2007CB411600), a special grant from the State Forestry Administration of China (2130206) and the Fundamental Research Funds for the Central Universities of China.

1 Frankham R, Ballou J D, Briscoe D A. Introduction to Conservation Genetics. New York: Cambridge University Press, 2002

2 Howard J G, Donoghue A M, Barone M A, et al. Successful induction of ovarian activity and laparoscopic intrauterine artificial insemination in the Cheetah (Acinonyx jubatus). J Zoo Wildl Med, 1992, 23: $288-300$

3 Wolf K N, Wildt D E, Vargas A, et al. Age dependent changes in sperm production, semen quality and testicular volume in the blackfooted ferret (Mustela nigripes). Biol Reprod, 2000, 63: 179-187

4 Ungerfeld R, González-Pensado S, Bielli A, et al. Reproductive biology of the pampas deer (Ozotoceros bezoarticus): A review. Acta Vet Scand, 2008, 50: 16

5 Andrabi S M, Maxwell W M. A review on reproductive biotechnologies for conservation of endangered mammalian species. Anim Reprod Sci, 2007, 99: 223-243

6 Xie Z, Gipps J. The 2010 International Studbook for Giant Panda (Ailuropoda melanoleuca). Beijing: Chinese Association of Zoological Garden, 2010

7 State Forestry Administration of China. The Third National Survey Report on Giant Panda in China. Beijing: Science Press, 2006

8 Wan Q H, Yu J Q, Yong Y G, et al. Reliable genetic analyses based on formalin-fixed materials: A new noninvasive sampling perspective for the giant panda. Conserv Genet, 2006, 7: 467-471
9 Brock M K, Whiteo B N. Application of DNA fingerprinting to the recovery program of the endangered Puerto Rican parrot. Proc Natl Acad Sci USA, 1992, 89: 11121-11125

10 Questiau S, Eybert M C, Taberlet P. Amplified fragment length polymorphism (AFLP) markers reveal extra-pair parentage in a bird species: The bluethroat (Luscinia svecica). Mol Ecol, 1999, 8: 13311339

11 Huang C W, Cheng Y S, Rouvier R, et al. AFLP fingerprinting for paternity testing in ducks. Br Poul Sci, 2007, 48: 323-330

12 Reis S P, Gonçalves E C, Silva A, et al. Genetic variability and efficiency of DNA microsatellite markers for paternity testing in horse breeds from the Brazilian Marajó archipelago. Genet Mol Biol, 2008, 31: 68-72

13 Paredes M, Norambuena M C, Molina B. Genetic diversity analysis in 12 microsatellite loci, used in equine paternity test in Chile. Arch Zoo, 2009, 58: 111-116

14 Stevanovic J, Stanimirovic Z, Dimitrijevic V, et al. Evaluation of 11 microsatellite loci for their use in paternity testing in Yugoslav Pied cattle (YU Simmental cattle). Czech J Anim Sci, 2010, 55: 221-226

15 Wan Q H, Wu H, Fujihara T, et al. Which genetic marker for which conservation genetic issue? Electrophoresis, 2004, 25: 2165-2176

16 Zhang H M, Guo Y, Li D S, et al. Sixteen novel microsatellite loci developed for the giant panda (Ailuropoda melanoleuca). Conserv Genet, 2009, 10: 589-592

17 Zane L, Bargelloni L, Patarnello T. Strategies for microsatellite isolation: A review. Mol Ecol, 2002, 11: 1-16

18 Sambrook J, Russell D W. Molecular Cloning: A Laboratory Manual, 3rd ed. Cold Spring Harbor: Cold Spring Harbor Laboratory Press, 2001

19 Belkhir K P, Borsa P, Chikhi L, et al. GENETIX, logiciel sous Windows $^{\mathrm{TM}}$ pour la génétique des populations. Montpellier: Laboratoire Génome, Populations, Interactions CNRS UMR 5000, Universitéde Montpellier II, 2001

20 Raymond M, Rousset F. GENEPOP (version 1.2): Population genetics software for exact tests and ecumenicism. J Hered, 1995, 86: 248-249

21 Lancaster A, Nelson M P, Meyer D, et al. PyPop: A software framework for population genomics: Analyzing large-scale multi-locus genotype data. In: Altman R B, Dunker A K, Hunter L, et al., eds. Pacific Symposium on Biocomputing 8 Singapore: World Scientific, 2003. 514-525

22 Lancaster A, Single R M, Solberg O D, et al. PyPop update-A software pipeline for large-scale multilocus population genomics. Tissue Antigens, 2007, 69: 192-197

23 SPSS Inc. SPSS 16.0 for Windows, Chicago: SPSS Inc., 2007

24 Fang S G, Wan Q H, Wu H, et al. Conservation Genetics of the Giant Panda. Beijing: Science Press, 2008

25 Kalinowski S T, Taper M L, Marshall T C. Revising how the computer program CERVUS accommodates genotyping error increases success in paternity assignment. Mol Ecol, 2007, 16: 1099-1106

26 Gerber S, Chabrier P, Kremer A. FaMoz: A software for parentage analysis using dominant, codominant and uniparentally inherited markers. Mol Ecol Notes, 2003, 3: 479-481

27 Fung W K. User-friendly programs for easy calculations in paternity testing and kinship determinations. Forensic Sci Int, 2003, 136: 22-34

Open Access This article is distributed under the terms of the Creative Commons Attribution License which permits any use, distribution, and reproduction in any medium, provided the original author(s) and source are credited. 